\title{
ON THE SECOND DEFORMATION LEMMA
}

\author{
JEAN-Nö̈L CORVELLEC
}

Dedicated to Andrzej Granas, with gratitude

\begin{abstract}
In the framework of critical point theory for continuous functionals defined on metric spaces, we give a new, simpler proof of the socalled Second Deformation Lemma, a basic tool of Morse theory.
\end{abstract}

The purpose of this note is to provide a new approach to the so-called Second Deformation Lemma (according to the terminology of [3]), for continuous functionals defined on metric spaces: see Theorem 4, an extension of [5, Theorem 2.10]. Roughly speaking, the new idea is reminiscent of the one which is commonly used when dealing with smooth functionals defined on smooth manifolds (see [2], [18], [20], [22]), when a "normalized" (pseudo-)gradient vector field is employed, in order that the functional decrease at constant rate along the deformation, also when approaching critical points. More precisely, starting with the notion of weak slope [10] and with the basic deformation result of [9], this is achieved by applying a variant (Theorem 2) of the Noncritical Interval Theorem of [1], [9], with a suitable choice of metric on the underlying space, thanks to a variant (Theorem 3) of a general change-of-metric procedure established in [6].

The point of view adopted here is technically simpler than the one in [5], as well as it sheds a new light on the result. As a matter of fact, the most technical parts in [5] borrowed much from earlier arguments in smooth settings. Compare also with the variant given in [15] of the basic result of [5], on deformation near an isolated critical point.

2000 Mathematics Subject Classification. 58E05.

Key words and phrases. Metric critical point theory, deformation theorems. 
This version of the Second Deformation Lemma allows to develop Morse theory for continuous functionals, as explicited in [5]. An application to a class of quasilinear problems is given in [8], where the associated functional is continuous, but not locally Lipschitzian in general. See also [1] for an overview of the connections between the abstract theory and problems of the calculus of variations.

In the sequel, $X$ is a metric space endowed with the metric $d$, and $f: X \rightarrow \mathbb{R}$ is a continuous function.

If $Y$ is a subset of $X$, a deformation of $Y$ is a continuous map $\eta: Y \times[0,1] \rightarrow Y$ such that $\eta(u, 0)=u$ for every $u \in Y$. If $Z$ is another subset of $X$, with $Z \subset Y$, the set $Z$ is a strong deformation retract of $Y$ if there exists a deformation $\eta$ of $Y$ such that $\eta(Y, 1) \subset Z$ and $\eta(u, t)=u$ for every $u \in Z$ and $t \in[0,1]$; the set $Z$ is a weak deformation retract of $Y$ if there exists a continuous $r: Y \rightarrow Z$, a deformation $\eta_{1}$ of $Y$, and a deformation $\eta_{2}$ of $Z$, such that $\eta_{1}(u, 1)=r(u)$ for $u \in Y$ and $\eta_{2}(v, 1)=r(v)$ for $v \in Z$ (see, e.g. [12]). In particular, $Z$ is a weak deformation retract of $Y$ if there exists a deformation $\eta$ of $Y$ with $\eta(Y, 1) \subset Z$ and $\eta(Z \times[0,1]) \subset Z$. Of course, a strong deformation retract is a weak one. We further observe, for later use, that if $Y$ is an ANR, if $\eta$ is a deformation of $Y$, and if $Z$ is a subset of $Y$ such that $\eta(Y \times] 0,1]) \subset Z$ (in particular, $Z$ is a weak deformation retract of $Y$ ), then $Z$ also is an ANR: this readily follows from a result of Hanner, see, e.g. [13, Theorem IV.6.3].

We shall denote by $B(u ; \delta)$ the open ball of radius $\delta>0$ centered at $u \in X$. If $Y$ and $Z$ are subsets of $X$, we set

$$
d(Y, Z):=\inf \{d(y, z): y \in Y, z \in Z\},
$$

with the convention that $d(Y, \emptyset)=\infty$. For $a \in \mathbb{R} \cup\{\infty\}$, we let

$$
f^{a}:=\{u \in X: f(u)<a\}, \quad \bar{f}^{a}:=\{u \in X: f(u) \leq a\} .
$$

We recall the notion of weak slope from [10, Definition (2.1)].

Definition 1. For $u \in X$ we denote by $|d f|(u)$ the supremum of the $\sigma$ 's in $[0, \infty[$ such that there exist $\delta>0$ and $\mathcal{H}: B(u ; \delta) \times[0, \delta] \rightarrow X$ continuous with

$$
d(\mathcal{H}(v, t), v) \leq t, \quad f(\mathcal{H}(v, t)) \leq f(v)-\sigma t .
$$

The extended real number $|d f|(u)$ is called the weak slope of $f$ at $u$. We say that $u \in X$ is a critical point of $f$ if $|d f|(u)=0$.

Note that $u \mapsto|d f|(u)$ is lower semicontinuous, as readily follows from the definition, while the set $\{u \in X:|d f|(u)<\infty\}$ is dense in $X$, as follows from Ekeland's variational principle, see e.g. [10, Corollary 3.4].

The above notion was introduced independently in [16], while a variant was introduced in [14]. We recall that if $X$ is a Finsler manifold of class $C^{1}$, and 
if $f$ is of class $C^{1}$, then $|d f|(u)=\left\|f^{\prime}(u)\right\|$ for $u \in X$. Observe that in this case, namely, if $X$ is of class $C^{1}$ only, then the classical deformation techniques of critical point theory do not apply, since only a continuous pseudo-gradient vector field for $f$ is available. Using the notion of weak slope, the following is the first global deformation result proved in [9, Theorem (2.8)]; here, we added to the statement some features which are both clear from the proof, and needed for our present purposes.

Theorem 1. Let $X$ be a metric space, $f: X \rightarrow \mathbb{R}$ and $\sigma: X \rightarrow[0, \infty[$ be two continuous functions such that

$$
|d f|(u) \neq 0 \Rightarrow|d f|(u)>\sigma(u)>0 .
$$

Then, there exist a continuous function $\tau: X \rightarrow[0, \infty[$ such that

$$
\tau(u)=0 \Leftrightarrow|d f|(u)=0,
$$

and a continuous map $\eta: X \times[0, \infty[\rightarrow X$ such that

(a) $d(\eta(u, t), u) \leq t$,

(b) $t \geq \tau(u) \Rightarrow \eta(u, t)=\eta(u, \tau(u))$,

(c) $0 \leq t \leq \tau(u) \Rightarrow f(\eta(u, t)) \leq f(u)-\sigma(u) t$.

(In particular, $\eta(u, t)=u$ for every $t$ if and only if $|d f|(u)=0$.)

Given $f: X \rightarrow \mathbb{R}$ continuous and $a \in \mathbb{R}$, we let

$$
K_{a}:=\{u \in X:|d f|(u)=0, f(u)=a\}
$$

denote the set of critical points of $f$ at the level $a$.

Corollary. Let $X$ be a metric space, $f: X \rightarrow \mathbb{R}$ be a continuous function, and $a \in \mathbb{R}$. Then, there exists a deformation $\eta: X \times[0,1] \rightarrow X$ such that

(a) $f(\eta(u, t)) \leq f(u)$,

(b) $|d f|(u)=0 \Rightarrow \eta(u, t)=u$,

(c) $u \in \bar{f}^{a}$ and $t>0 \Rightarrow \eta(u, 1) \in f^{a} \cup K_{a}$.

In particular, $f^{a} \cup K_{a}$ is a weak deformation retract of $\bar{f}^{a}$.

Proof. Since $|d f|$ is lower semicontinuous and not identically equal to $\infty$, letting

$$
\sigma(u):=\frac{1}{2} \inf \{d(u, v)+|d f|(v): v \in X\},
$$

we have that $\sigma: X \rightarrow[0, \infty[$ is a continuous function with $\sigma(u)=0$ if $|d f|(u)=0$, and

$$
|d f|(u) \neq 0 \Rightarrow|d f|(u)>\sigma(u)>0 .
$$

Let then $\eta^{\prime}: X \times[0, \infty[\rightarrow X$ and $\tau: X \rightarrow[0, \infty[$ be the continuous map and function given by Theorem 1 . It is readily seen that the map $\eta: X \times[0,1] \rightarrow X$ defined by $\eta(u, t):=\eta^{\prime}(u, \tau(u) t)$ has the required properties. 
REMARK 1. According to a previous observation, the above corollary shows that if $\bar{f}^{a}$ is an ANR, then so is $f^{a} \cup K_{a}$. We shall comment more on this matter in our final remarks.

The next result also builds upon Theorem 1.

TheOREM 2. Let $X$ be a metric space, $f: X \rightarrow \mathbb{R}$ be a continuous function, $a, c \in \mathbb{R}$ with $a<c$, and $\sigma>0$. Assume that $f^{-1}([a, c])$ is complete, and that

$$
\left.\left.|d f|(u)>\sigma \quad \text { for every } u \in f^{-1}(] a, c\right]\right) .
$$

Then, there is a deformation $\eta: \bar{f}^{c} \times[0,1] \rightarrow \bar{f}^{c}$ such that:

(a) $f(\eta(u, t)) \leq f(u)$,

(b) $u \in \bar{f}^{a} \Rightarrow \eta(u, t)=u$,

(c) $\left.\eta\left(\bar{f}^{c}, 1\right)\right) \subset \bar{f}^{a}$.

Thus, $\bar{f}^{a}$ is a strong deformation retract of $\bar{f}^{c}$.

Proof. Set $\left.\left.U:=f^{-1}(] a, c\right]\right)$, and let $\eta_{1}: U \times\left[0, \infty\left[\rightarrow U\right.\right.$ and $\tau_{1}: U \rightarrow$ ] $0, \infty[$, both continuous, be given by Theorem 1 , such that for every $(u, t) \in$ $U \times[0, \infty[$ we have

$$
\begin{gathered}
d\left(\eta_{1}(u, t), u\right) \leq t, \quad f\left(\eta_{1}(u, t)\right) \leq f(u), \\
t \geq \tau_{1}(u) \Rightarrow \eta_{1}(u, t)=\eta_{1}\left(u, \tau_{1}(u)\right), \\
0 \leq t \leq \tau_{1}(u) \Rightarrow f\left(\eta_{1}(u, t)\right) \leq f(u)-\sigma t .
\end{gathered}
$$

For $h \in \mathbb{N}, h \geq 2$, define recursively (as in the proof of [9, Theorem (2.11)], for $(u, t) \in U \times[0, \infty[:$

$$
\begin{gathered}
\eta_{h}(u, t)= \begin{cases}\eta_{h-1}(u, t) & \text { if } 0 \leq t \leq \tau_{h-1}(u), \\
\eta_{1}\left(\eta_{h-1}\left(u, \tau_{h-1}(u)\right), t-\tau_{h-1}(u)\right) & \text { if } t \geq \tau_{h-1}(u)\end{cases} \\
\tau_{h}(u)=\tau_{h-1}(u)+\tau_{1}\left(\eta_{h-1}\left(u, \tau_{h-1}(u)\right)\right)
\end{gathered}
$$

Clearly, each $\eta_{h}$ is continuous and, for every $u,\left(\tau_{h}(u)\right)$ is an increasing sequence of positive real numbers. For each $u \in U$ and each $h \in \mathbb{N}$, we have that

$$
0 \leq t \leq \tau_{h}(u) \Rightarrow f\left(\eta_{h}(u, t)\right) \leq f(u)-\sigma t,
$$

and

$$
d\left(\eta_{h+1}\left(u, \tau_{h+1}(u)\right), \eta_{h}\left(u, \tau_{h}(u)\right)\right) \leq \tau_{h+1}(u)-\tau_{h}(u)=\tau_{1}\left(\eta_{h}\left(u, \tau_{h}(u)\right)\right) .
$$

We infer from $(2)$ that $\tau_{h}(u) \leq(f(u)-a) / \sigma$ for each $h \in \mathbb{N}$, hence there exists $\left.t(u):=\lim _{h} \tau_{h}(u) \in\right] 0,(f(u)-a) \sigma\left[\right.$. We then obtain from $(3)$ that $\left(\eta_{h}\left(u, \tau_{h}(u)\right)\right)$ is a Cauchy sequence in $\left.\left.f^{-1}(] a, c\right]\right)$. Hence, letting $\bar{u}:=\lim _{h} \eta_{h}\left(u, \tau_{h}(u)\right)$, we see that $f(\bar{u})=a$ - for, otherwise, we have $a<f(\bar{u})<c$ while $\tau_{1}(\bar{u})=0$, which is not true. 
For $(u, t) \in \bar{f}^{c} \times[0,1]$, set:

$$
\eta(u, t):= \begin{cases}\lim _{h \rightarrow \infty} \eta_{h}(u, t t(u)) & \text { if } f(u)>a, \\ u & \text { if } f(u) \leq a .\end{cases}
$$

Let $u \in U$. If $t \in\left[0,1\left[\right.\right.$, let $k \in \mathbb{N}$ be such that $\tau_{k}(u) \geq t t(u)$, then $\eta_{h}(u, t t(u))=$ $\eta_{k}(u, t t(u))$ for $h \geq k$. On the other hand,

$$
\lim _{h \rightarrow \infty} \eta_{h}(u, t(u))=\lim _{h \rightarrow \infty} \eta_{h}\left(u, \tau_{h}(u)\right)=\bar{u},
$$

so that $\eta$ is well-defined. Furthermore, we clearly have $f(\eta(u, t)) \leq f(u)$ for every $(u, t) \in \bar{f}^{c} \times[0,1]$, while $\eta(\cdot, 1)$ maps $\bar{f}^{c}$ in $\bar{f}^{a}$, so that it remains to show that $\eta$ is continuous. It is clear that $\eta$ is continuous at $(u, t)$ if either $f(u)<a$, or $f(u)>a$ and $t<1$. Assume that $f(u)>a$ and $t=1$. Observe that for $v \in U$ and $h, k \in \mathbb{N}, k \geq h$, we have

$$
f\left(\eta_{k}\left(v, \tau_{k}(v)\right)\right)-f\left(\eta_{h}\left(v, \tau_{h}(v)\right)\right) \leq-\sigma\left(\tau_{k}(v)-\tau_{h}(v)\right) .
$$

This shows, first, that $v \mapsto t(v)$ is continuous at $u$ : indeed, let $\varepsilon>0$, and let $\gamma>0, \gamma \leq \varepsilon$, be such that $f(w) \leq a+\varepsilon \sigma$ for every $w \in B(\bar{u} ; \gamma)$. Let then $h \in \mathbb{N}$ and $\delta>0$ be such that

$$
\eta_{h}\left(v, \tau_{h}(v)\right) \in B(\bar{u} ; \gamma) \text { and }\left|\tau_{h}(v)-\tau_{h}(u)\right| \leq \varepsilon \quad \text { for every } v \in B(u ; \delta) \cap U .
$$

Let $v \in B(u ; \delta) \cap U$. It follows from (4) that $\tau_{k}(v)-\tau_{h}(v) \leq \varepsilon$ for every $k \geq h$, whence $t(v)-\tau_{h}(v) \leq \varepsilon$, so that

$$
|t(v)-t(u)| \leq t(v)-\tau_{h}(v)+\left|\tau_{h}(v)-\tau_{h}(u)\right|+t(u)-\tau_{h}(u) \leq 3 \varepsilon .
$$

We may thus have chosen (as we now do) $\delta$ so small that, also, $1-\delta \geq \tau_{h}(v) / t(v)$ for every $v \in B(u ; \delta) \cap U$. Then, if $v \in B(u ; \delta) \cap U$, if $t \in[1-\delta, 1]$, and if $k \geq h$, we have $t t(v) \geq \tau_{h}(v)$ and $\eta_{k}(v, t t(v))=\eta_{k}\left(v, \min \left\{\tau_{k}(v), t t(v)\right\}\right)$, so that

$$
\begin{aligned}
d\left(\eta_{k}(v, t t(v)), \bar{u}\right) & \leq d\left(\eta_{k}(v, t t(v)), \eta_{h}\left(v, \tau_{h}(v)\right)\right)+d\left(\eta_{h}\left(v, \tau_{h}(v)\right), \bar{u}\right) \\
& \leq \tau_{k}(v)-\tau_{h}(v)+\gamma \leq 2 \varepsilon
\end{aligned}
$$

and consequently, $d(\eta(v, t), \bar{u}) \leq 2 \varepsilon$, which shows that $\eta$ is continuous at $(u, 1)$. Arguing in a similar (but simpler) way, one shows that $\eta$ is also continuous at $(u, t)$ whenever $f(u)=a$.

The preceding result was partly motivated by [4, Lemma 4.1], where $X=\mathbb{R}^{n}$, $f$ is Lipschitz continuous and of class $C^{2}$ on a neighbourhood of $\left.\left.f^{-1}(] a, c\right]\right)$, and the proof standardly uses the flow generated by the negative (normalized) gradient of $f$ - a very special case of Theorem 2 , but the point here is that $f$ is implicitly allowed to possess critical points at the level $a$. Of course, whenever $X$ is, say, a Banach space and $f$ is of class $C^{1}$ on $X$, the fact that $K_{a}$ be nonempty is not compatible with assumption (1). Since, as usual, we want to cover this 
important case as well as others, we shall show how it can be treated, and to what extent, through an appropriate change of the metric $d$, according to the following result.

Theorem 3. Let $(X, d)$ be a metric space, $A$ be a nonempty subset of $X$, and $\beta:[0, \infty[\rightarrow[0, \infty[$ be a continuous function with $\beta(s)>0$ for $s>0$. Assume that either $\beta(0)>0$, or $A$ is compact and its connected components are single points. Then, there exists a metric $\widetilde{d}$ on $X$ which is topologically equivalent to $d$ and such that

(a) For any subset $B$ of $X$, we have

$$
\widetilde{d}(B, A) \geq \int_{0}^{d(B, A)} \beta(s) d s .
$$

Consequently, if $\int_{0}^{\infty} \beta(s) d s=\infty$, then $(X, \widetilde{d})$ is complete if $(X, d)$ is complete.

(b) Letting $\beta_{A}:=\{u \in X: \beta(d(u, A))=0\}$, if $f: X \rightarrow \mathbb{R}$ is a continuous function, and if we denote by $|\widetilde{d} f|$ the weak slope of $f$ with respect to the metric $\widetilde{d}$, we have

$$
|\widetilde{d} f|(u)=\frac{|d f|(u)}{\beta(d(u, A))} \quad \text { for every } u \in X \backslash \beta_{A} .
$$

Proof. The result is proved in [6, Theorem 4.1] in the case when $\beta$ has positive values (that is, $\beta_{A}=\emptyset$ ). We thus assume that $\beta(0)=0$ (so that $\left.\beta_{A}=A\right), A$ is compact, and the connected components of $A$ are single points.

The metric $\widetilde{d}$ is defined as follows: let $Y$ be a Banach space such that $X$ is isometrically embedded in $Y$, and, for $u, v \in Y$, denote by $\Gamma_{u, v}$ the set of $C^{1}$-paths $\gamma:[0,1] \rightarrow Y$ with $\gamma(0)=u, \gamma(1)=v$; we set

$$
\widetilde{d}(u, v):=\inf _{\gamma \in \Gamma_{u, v}} \int_{0}^{1} \beta(d(\gamma(s), A))\left\|\gamma^{\prime}(s)\right\| d s,
$$

where $d$ also stands for the distance associated to the norm. The arguments in the proof of [6, Theorem 4.1] readily yield all the assertions made, but for the following points, for which some refinements are needed: $\widetilde{d}(u, v)>0$ whenever $u \neq v$ (so that $\widetilde{d}$ indeed be a metric on $X$ ); $\widetilde{d}$-convergence implies $d$-convergence; and $(X, \widetilde{d})$ is complete if $\int_{0}^{\infty} \beta(s) d s=\infty$ and if $(X, d)$ is complete.

The first two points are true if we show that given $u \in Y$ and $r>0$, there exists $\delta>0$ such that

$$
\|u-v\| \geq r \Rightarrow \widetilde{d}(u, v) \geq \delta .
$$

This is clear if $u \notin A$, in fact simpler to prove than the case $u \in A$, that we shall treat. We may assume that $F:=X \backslash B(u ; r) \neq \emptyset$. From the assumptions on $A$, we find two disjoint compact sets $F_{1}$ and $F_{2}$ such that $u \in F_{1}, A \cap F \subset F_{2}$, and 
$A=F_{1} \cup F_{2}$. Indeed, if $A \cap F=\emptyset$, we take $F_{2}=\emptyset$; otherwise, use the chain argument, see, e.g. [17, 5.T]. Of course, $F_{1} \subset B(u ; r)$. Set

$$
\alpha:=\min \left\{\frac{1}{2} d\left(F_{1}, F\right), \frac{1}{4} d\left(F_{1}, F_{2}\right)\right\}>0 \quad \text { and } \quad \varepsilon:=\min \{\beta(s): s \in[\alpha, 2 \alpha]\} .
$$

Let $v \in F$ and $\gamma \in \Gamma_{u, v}$. By the choice of $\alpha$, we find $0<t_{\gamma}<t_{\gamma}^{\prime} \leq 1$ such that

$$
d\left(\gamma\left(t_{\gamma}\right), A\right)=\alpha, \quad d\left(\gamma\left(t_{\gamma}^{\prime}\right), A\right)=2 \alpha, \quad \text { and } \quad d(\gamma(s), A) \in[\alpha, 2 \alpha]
$$

for all $s \in\left[t_{\gamma}, t_{\gamma}^{\prime}\right]$ (consider the cases $d\left(\gamma([0,1]), F_{2}\right) \geq 2 \alpha, d\left(\gamma([0,1]), F_{2}\right)<2 \alpha$ whenever $F_{2} \neq \emptyset$ ). We thus have

$$
\begin{aligned}
\int_{0}^{1} \beta(d(\gamma(s), A))\left\|\gamma^{\prime}(s)\right\| d s & \geq \int_{t_{\gamma}}^{t_{\gamma}^{\prime}} \beta(d(\gamma(s), A))\left\|\gamma^{\prime}(s)\right\| d s \\
& \geq \varepsilon\left\|\gamma\left(t_{\gamma}^{\prime}\right)-\gamma\left(t_{\gamma}\right)\right\| \geq \varepsilon \alpha,
\end{aligned}
$$

so that $\widetilde{d}(u, v) \geq \varepsilon \alpha=: \delta$.

We now show that for any $0<r<R$ there exists $\delta>0$ such that

$$
r \leq d\left(u_{i}, A\right) \leq R, i=1,2 \Rightarrow \widetilde{d}\left(u_{1}, u_{2}\right) \geq \delta \min \left\{r / 2, R,\left\|u_{1}-u_{2}\right\|\right\} .
$$

Indeed, set $\delta:=\min \{\beta(s): s \in[r / 2,2 R]\}$, and for $\gamma \in \Gamma_{u_{1}, u_{2}}$, let

$$
\left.\left.t_{\gamma}:=\sup \{t \in[0,1]: r / 2 \leq d(\gamma(s), A) \leq 2 R \quad \text { for all } s \in[0, t]\} \in\right] 0,1\right] .
$$

Then,

$$
\int_{0}^{t_{\gamma}} \beta(d(\gamma(s), A))\left\|\gamma^{\prime}(s)\right\| d s \geq \delta\left\|u_{1}-\gamma\left(t_{\gamma}\right)\right\| \geq \delta \min \left\{r / 2, R,\left\|u_{1}-u_{2}\right\|\right\},
$$

considering the cases $t_{\gamma}<1$ (so that either $d\left(\gamma\left(t_{\gamma}\right), A\right)=r / 2$, or $d\left(\gamma\left(t_{\gamma}\right), A\right)=$ $2 R$ ), and $t_{\gamma}=1$. Since $\gamma$ is arbitrary in $\Gamma_{u_{1}, u_{2}},(6)$ follows.

Assume that $\int_{0}^{\infty} \beta(s) d s=\infty$, and let $\left(u_{h}\right)$ be a Cauchy sequence in $(X, \widetilde{d})$. According to (5), the sequence $\left(d\left(u_{h}, A\right)\right)$ is bounded, and in particular, we have that $\rho:=\liminf _{h \rightarrow \infty} d\left(u_{h}, A\right)$ is finite. If $\rho=0$, there exist a subsequence $\left(v_{h}\right)$ of $\left(u_{h}\right)$ and a point $u \in A$ such that $\left(v_{h}\right) d$-converges, hence $\widetilde{d}$-converges, to $u$, so that $\left(u_{h}\right) \widetilde{d}$-converges to $u$. If $\rho>0$, we find $0<r<R$ such that $r \leq d\left(u_{h}, A\right) \leq R$ for $h$ large enough, and it follows from (6) that $\left(u_{h}\right)$ is a Cauchy sequence in $(X, d)$. Thus, $(X, \widetilde{d})$ is complete if $(X, d)$ is complete.

The verification of assumption (1) in Theorem 2 depends, in practice, on the verification of a Palais-Smale type condition, a version of which we now recall.

Definition 2. Let $X$ be a metric space, $f: X \rightarrow \mathbb{R}$ be a continuous function, and $U$ a nonempty closed subset of $X$. We say that a sequence $\left(u_{h}\right) \subset U$ is a Palais-Smale sequence for $f$ in $U$ (a (PS) sequence for $f$ in $U$, for short), if the sequence $\left(|d f|\left(u_{h}\right)\right)$ converges to 0 and the sequence $\left(f\left(u_{h}\right)\right)$ is bounded. 
We say that $f$ satisfies the Palais-Smale condition in $U$ (condition (PS) in $U$, for short), if every (PS) sequence for $f$ in $U$ contains a subsequence converging in $X$. Note that since $|d f|$ is lower semicontinuous and $U$ is closed, any cluster point of a (PS) sequence for $f$ in $U$ is a critical point of $f$ in $U$.

The connection between the (PS) condition and Theorem 3 is established through the following

Lemma. Let $X$ be a metric space, $f: X \rightarrow \mathbb{R}$ be a continuous function, $U$ be a closed subset of $X$ with nonempty interior, and let $K_{U}$ denote the (possibly empty) set of critical points of $f$ in $U$. Assume that $f$ is bounded on $U$ and satisfies condition (PS) in $U$. Then, $K_{U}$ is compact and there exists a continuous nondecreasing function $\beta:[0, \infty[\rightarrow[0, \infty[$ such that $\beta(s)>0$ if $s>0$, and

$$
|d f|(u)>\beta\left(d\left(u, K_{U}\right)\right) \quad \text { for every } u \in U \backslash K_{U} .
$$

Proof. The fact that $K_{U}$ is compact readily follows from the (PS) condition and the boundedness of $f$ on $U$. For $s \geq 0$, set

$$
\widetilde{\beta}(s):=\frac{1}{2} \inf \left\{|d f|(u): u \in U, \text { and } d\left(u, K_{U}\right) \geq s \text { whenever } K_{U} \neq \emptyset\right\},
$$

then $\widetilde{\beta}$ is real-valued and nondecreasing. Also, $\widetilde{\beta}(s)>0$ if $s>0$ - for, otherwise, we could find a (PS) sequence for $f$ in $U$ at positive distance of $K_{U}$, in contradiction with the fact that $f$ satisfies the (PS) condition in $U$. Thus, $\widetilde{\beta}$ is a positive constant if $K_{U}=\emptyset$, in which case we are done. So, we now assume that $K_{U} \neq \emptyset$, in which case $\widetilde{\beta}(s)=0$ if and only if $s=0$. Also, if $u \notin K_{U}$ then $|d f|(u)>\widetilde{\beta}\left(d\left(u, K_{U}\right)\right)$. It is easily seen that the lower semicontinuous regularization $\widehat{\beta}$ of $\widetilde{\beta}$ has the same mentioned properties. If we now set

$$
\beta(s):=\inf \{|s-\tau|+\widehat{\beta}(\tau): \tau \geq 0\}
$$

then, $\beta$ is continuous, and also possesses the required properties.

A notion of modulus of regularity of a function $f$ on an open subset $G \subset X$ with respect to a disjoint closed subset $C \subset X$ is given in [14], which is a function with similar properties as $\beta$ in the Lemma, see [14, Definition 3], and the remark that follows it, concerning an early contribution of Morse [20] in this direction.

We finally state our version of the Second Deformation Lemma.

Theorem 4. Let $X$ be a metric space, $f: X \rightarrow \mathbb{R}$ be a continuous function, and $a \in \mathbb{R}, b \in \mathbb{R} \cup\{\infty\}$ with $a<b$. Assume that for every $c \in\left[a, b\left[, f^{-1}([a, c])\right.\right.$ is complete and $f$ satisfies condition (PS) in $f^{-1}([a, c])$, that $f$ has no critical point $u$ with $a<f(u)<b$, and that either $K_{a}=\emptyset$ or the connected components 
of $K_{a}$ are single points. Then, there exists a deformation $\eta: f^{b} \times[0,1] \rightarrow f^{b}$ such that
(a) $f(\eta(u, t)) \leq f(u)$
(b) $u \in K_{a} \Rightarrow \eta(u, t)=u$,
(c) $\eta\left(f^{b}, 1\right) \subset f^{a} \cup K_{a}$.

In particular, $f^{a} \cup K_{a}$ is a weak deformation retract of $f^{b}$.

Proof. We may of course assume that $f^{-1}(] a, b[) \neq \emptyset$, and, further, that $K_{a} \neq \emptyset$ (the case $K_{a}=\emptyset$ is just simpler - not requiring Theorem 3).

Let $\left(c_{h}\right)$ be an increasing sequence of real numbers with $c_{1}>a$ and $\lim _{h} c_{h}=$ $b$, and let $c_{0}:=a$. Thanks to the (PS) condition, to the fact that $f$ has no critical point in $f^{-1}(] a, b[)$, and to the Lemma, we find a sequence of positive real numbers $\left(\sigma_{h}\right)_{h \geq 2}$ and a continuous nondecreasing function $\beta:[0, \infty[\rightarrow[0, \infty[$ with $\beta(s)>0$ for $s>0$, such that

$$
\begin{array}{ll}
|d f|(u)>\sigma_{h} & \text { for every } u \in f^{-1}\left(\left[c_{h-1}, c_{h+1}\right]\right), h \geq 2, \\
|d f|(u)>\beta\left(d\left(u, K_{a}\right)\right) & \text { for every } u \in f^{-1}\left(\left[a, c_{2}\right]\right) \backslash K_{a} .
\end{array}
$$

Recall also that $K_{a}$ is compact. We consider $X$ as endowed with the metric $\widetilde{d}$ associated to $\beta$ and $A:=K_{a}=\beta_{A}$, according to Theorem 3: then, $f$ is continuous on $(X, \widetilde{d}), f^{-1}\left(\left[a, c_{2}\right]\right)$ is complete in $(X, \widetilde{d})$, and

$$
|\widetilde{d} f|(u)=\frac{|d f|(u)}{\beta\left(d\left(u, K_{a}\right)\right)}>1 \quad \text { for every } u \in f^{-1}\left(\left[a, c_{2}\right]\right) \backslash K_{a},
$$

according to (8), where $|\widetilde{d} f|$ denotes the weak slope with respect to $\widetilde{d}$. In view of (7), (9), and applying Theorem 2 in $(X, d)$ for $h \geq 2$ and in $(X, \widetilde{d})$ for $h=1$, we find deformations $\eta_{h}^{\prime}: \bar{f}^{c_{h+1}} \times[0,1] \rightarrow \bar{f}^{c_{h+1}}$ such that

$$
\begin{gathered}
f\left(\eta_{h}^{\prime}(u, t)\right) \leq f(u), \quad f\left(\eta_{h}^{\prime}(u, 1)\right) \leq c_{h-1}, \\
f(u) \leq c_{h-1} \Rightarrow \eta_{h}^{\prime}(u, t)=u .
\end{gathered}
$$

Observe that $\eta_{1}^{\prime}$ is continuous with respect to the metric $d$ as well as to the metric $\widetilde{d}$, and from now on till the end of the proof, we stay in $(X, d)$.

We now construct, as in the proof of the Noncritical Interval Theorem $[9$, Theorem (2.15)], a strong deformation retraction $\eta^{\prime}$ of $f^{b}$ in $\bar{f}^{a}$. Let $\vartheta: \mathbb{R} \rightarrow[0,1]$ be defined by $\vartheta(s)=0$ for $s \leq 0, \vartheta(s)=s$ for $s \in[0,1]$, and $\vartheta(s)=1$ for $s \geq 1$. For each $h \in \mathbb{N}$, define a deformation $\eta_{h}^{\prime \prime}: X \times[0,1] \rightarrow X$ by

$$
\eta_{h}^{\prime \prime}(u, t):=\eta_{h}^{\prime}\left(u, \vartheta\left(\frac{c_{h+1}-f(u)}{c_{h+1}-c_{h}}\right) t\right)
$$


so that

$$
\begin{gathered}
f\left(\eta_{h}^{\prime \prime}(u, t)\right) \leq f(u), \\
f(u) \leq c_{h} \Rightarrow f\left(\eta_{h}^{\prime \prime}(u, 1)\right) \leq c_{h-1}, \quad f(u) \leq c_{h-1} \Rightarrow \eta_{h}^{\prime \prime}(u, t)=u .
\end{gathered}
$$

Define $\eta_{h}: X \times[0,1] \rightarrow X$ by

$$
\begin{aligned}
\eta_{1}(u, t) & :=\eta_{1}^{\prime \prime}(u, t), \\
\eta_{h}(u, t) & :=\eta_{h-1}\left(\eta_{h}^{\prime \prime}(u, t), t\right), \quad h \geq 2 .
\end{aligned}
$$

Arguing by induction, it is easy to see that, for each $h$,

$$
\begin{gathered}
\eta_{h}(u, 0)=u, \quad f\left(\eta_{h}(u, t)\right) \leq f(u), \\
f(u) \leq c_{h} \Rightarrow f\left(\eta_{h}(u, 1)\right) \leq a, \\
f(u) \leq c_{h-1} \Rightarrow \eta_{h}(u, t)=\eta_{h-1}(u, t), \quad h \geq 2, \\
f(u) \leq a \Rightarrow \eta_{h}(u, t)=u,
\end{gathered}
$$

so that the map $\eta^{\prime}: f^{b} \times[0,1] \rightarrow f^{b}$ defined by $\eta^{\prime}(u, t):=\lim _{h \rightarrow \infty} \eta_{h}(u, t)$ is a strong deformation retraction of $f^{b}$ in $\bar{f}^{a}$, and satisfies (a).

Finally, let $\eta^{\prime \prime}: X \times[0,1] \rightarrow X$ be given by the Corollary, and providing a weak deformation retraction of $\bar{f}^{a}$ in $f^{a} \cup K_{a}$. Then, the map $\eta: f^{b} \times[0,1] \rightarrow f^{b}$ defined by $\left.\eta(u, t):=\eta^{\prime \prime}\left(\eta^{\prime}(u, t), t\right)\right)$ has the required properties.

Remarks 2. (i) Theorem 4 extends [5, Theorem 2.10], where it is assumed that $K_{a}$ is a finite set. The more general assumption on $K_{a}$ made here is the same as in [3, Theorem I.3.2] (where $X$ is a $C^{2}$ Finsler manifold and $f$ is of class $C^{1}$ ), and it allows, for example, $K_{a}$ to be an infinite sequence together with its limit. In view of Theorem 3 , this assumption on $K_{a}$ seems to be the weakest possible, in general. On the other hand, $K_{a}$ might be any set in particular settings, meaning that Theorem 2 can be used directly, without changing the metric: recall the comments after Theorem 2 .

(ii) In [5, Theorem 2.10], it was written by mistake that the deformation $\eta$ was a deformation of the whole space $X$, which is (clearly) not the case, in general, if $b \in \mathbb{R}$ and $K_{b} \neq \emptyset$. Of course, this mistake does not affect the validity of the other results in [5], which are just based on the fact that $f^{a} \cup K_{a}$ is a weak deformation retract of $f^{b}$. On the contrary, if $b \in \mathbb{R}$ and $K_{b}=\emptyset$, then the deformation $\eta$ can be extended to $X \times[0,1]$, and the conclusion (c) strengthened to read

$$
f(u) \leq b \Rightarrow \eta(u, 1) \in f^{a} \cup K_{a} .
$$

This can be seen making further use of the Corollary at the level $b$, as in the proof of the Noncritical Interval Theorem [1, Theorem 1.1.14], whence yielding an extension of the latter result. 
(iii) As evoked at the beginning of this note, Theorem 4 allows to develop Morse theory for continuous functionals through the notion of critical groups, which are defined in [5] using relative singular homology (as is usual). Results on computation of the critical groups for a class of continuous functionals associated with quasilinear elliptic problems are established in [8]. These results essentially deal with a nondegenerate situation (in a suitable sense); under appropriate assumptions, also a degenerate situation is treated in [18], through a nonsmooth adaptation of the Generalized Morse Lemma of [12] in a neighbourhood of an isolated critical point. In [18], the critical groups are defined via Alexander-Spanier cohomology, since use is made, as a substitute for Theorem 4, of [11, Theorem 2.7]. The latter result shows that, letting $K_{a}$ be any set in Theorem 4 , the inclusion $\bar{f}^{a} \subset f^{b}$ still induces an isomorphism $H^{*}\left(\bar{f}^{a}\right) \rightarrow H^{*}\left(f^{b}\right)$ for Alexander-Spanier cohomology. Indeed, thanks to the tautness property of Alexander-Spanier cohomology, it suffices to use the nonsmooth version of the First Deformation Lemma given in [9, Theorem (2.14)], in order to deal with the (possibly) critical level $a$.

In a general metric space, the critical groups depend on the particular choice of the (co)homology theory involved in the definition. I owe to Marco Degiovanni the suggestion that it may be worth noticing that if the metric space $X$ is an ANR, then Theorem 4 shows that so are $f^{a} \cup K_{a}$ and $\bar{f}^{a}$. Indeed, we mentioned in the proof that $\bar{f}^{a}$ is a strong deformation retract of $f^{b}$, hence an ANR; then, the fact that also $f^{a} \cup K_{a}$ is an ANR is already observed in Remark 1. In this situation, all the "usual" cohomology theories agree, and so do the critical groups.

(iv) The change-of-metric procedure (Theorem 3) can be used for various purposes (leading to applications in existence results for partial differential equations), also in situations when the set $A$ is not a set of critical points of a given functional, see [6], [7] for some examples.

\section{REFERENCES}

[1] A. Canino and M. Degiovanni, Nonsmooth critical point theory and quasilinear elliptic equations, Topological Methods in Differential Equations and Inclusions (Montréal, 1994) (A. Granas and M. Frigon, eds.), pp. 1-50; NATO Adv. Sci. Inst. Ser. C 472 (1995).

[2] K. C. Chang, Solutions of asymptotically linear operator equations via Morse theory, Comm. Pure Appl. Math. 34 (1981), 693-712.

[3] K. C. Chang, Infinite Dimensional Morse Theory and Multiple Solution Problems, Birkhäuser, Boston, 1993.

[4] B. Cornet and M.-O. Czarnecki, Existence of generalized equilibria, Nonlinear Anal. 44 (2001), 555-573.

[5] J.-N. Convellec, Morse theory for continuous functionals, J. Math. Anal. Appl. 195 (1995), 1050-1072. 
[6] Quantitative deformation theorems and critical point theory, Pacific J. Math. 187 (1999), 263-279.

[7] - On critical point theory with the (PS)* condition, Calculus of Variations and Differential Equations (A. Ioffe, S. Reich and I. Shafrir, eds.), Haifa, 1998, pp. 65-81; Chapman \& Hall/CRC Research Notes in Math. Series, vol. 410, CRC Press, Boca Raton, Fla, 2000.

[8] J.-N. Corvellec and M. Degiovanni, Nontrivial solutions of quasilinear equations via nonsmooth Morse theory, J. Differential Equations 136 (1997), 268-293.

[9] J.-N. Corvellec, M. Degiovanni and M. Marzocchi, Deformation properties for continuous functionals and critical point theory, Topol. Methods Nonlinear Anal. 1 (1993), 151-171.

[10] M. Degiovanni and M. Marzocchi, A critical point theory for nonsmooth functionals, Ann. Mat. Pura Appl. 167 (1994), 73-100.

[11] M. Degiovanni And L. Morbini, Closed geodesics with Lipschitz obstacle, J. Math. Anal. Appl. 233 (1999), 767-789.

[12] D. Gromoll and W. Meyer, On differentiable functions with isolated critical points, Topology 8 (1969), 361-369.

[13] S.-T. Hu, Theory of Retracts, Wayne State University Press, 1965.

[14] A. Ioffe And E. Schwartzman, Metric critical point theory 1. Morse regularity and homotopic stability of a minimum, J. Math. Pures Appl. 75 (1996), 125-153.

[15] Metric critical point theory 2. Deformation techniques, New Results in Operator Theory and its Applications, Oper. Theory Adv. Appl. 1996, Birkhäuser, Basel, 1997, pp. 131-144.

[16] G. KATRIEL, Mountain pass theorems and global homeomorphism theorems, Ann. Inst. H. Poincaré Anal. Non Linéaire 11 (1994), 189-209.

[17] J. L. Kelley, General Topology, Springer-Verlag, New York-Heidelberg-Berlin, 1955.

[18] S. LanCElotti, Morse index estimates for continuous functionals associated with quasilinear elliptic equations, preprint (1999).

[19] A. Marino and G. Prodi, Metodi perturbativi nella teoria di Morse, Boll. Un. Mat. Ital. 11 (1975), 1-32.

[20] M. Morse, Functional Topology and Abstract Variational Theory Mémorial des Sciences Mathématiques Fasc., vol. XCII, Gauthier-Villars, Paris, 1938.

[21] E. H. Rothe, Morse theory in Hilbert space, Rocky Mountain J. Math. 3 (1973), 251274.

[22] E. H. Spanier, Algebraic Topology, McGraw-Hill, New York, 1966.

[23] Z. Q. Wang, A note on the deformation theorem, Acta Math. Sinica 30 (1987), 106-110.

JEAN-NoËL CoRvelleC

Laboratoire de Modélisation

Analyse Non-linéaire et Optimisation (MANO)

Université de Perpignan

66860 Perpignan cedex, FRANCE

E-mail address: corvellec@univ-perp.fr 